\section{Massive cerebral infarction after completion pneumonectomy for pulmonary torsion}

\author{
J Hendriks, P Van Schil, W De Backer, \\ E Hauben, R Vanmaele, E Van Marck
}

\begin{abstract}
A case of lower lobe torsion after left upper lobectomy is reported which was complicated by a cerebral infarction after completion pneumonectomy. Results of a small survey in Flanders revealed two additional cases of pulmonary torsion after lobectomy.
\end{abstract}

(Thorax 1994;49:1274-1275)

Department of Pulmonary Medicine W De Backe

Department of Pathology

E Hauben

E Van Marck

University Hospital of Antwerp, B-2650 Edegem, Belgium

Reprint requests to: Dr P Van Schil.

Received 6 January 1994 Returned to author 17 February 1994 Revised version received 28 February 1994 Accepted for publication 2 March 1994

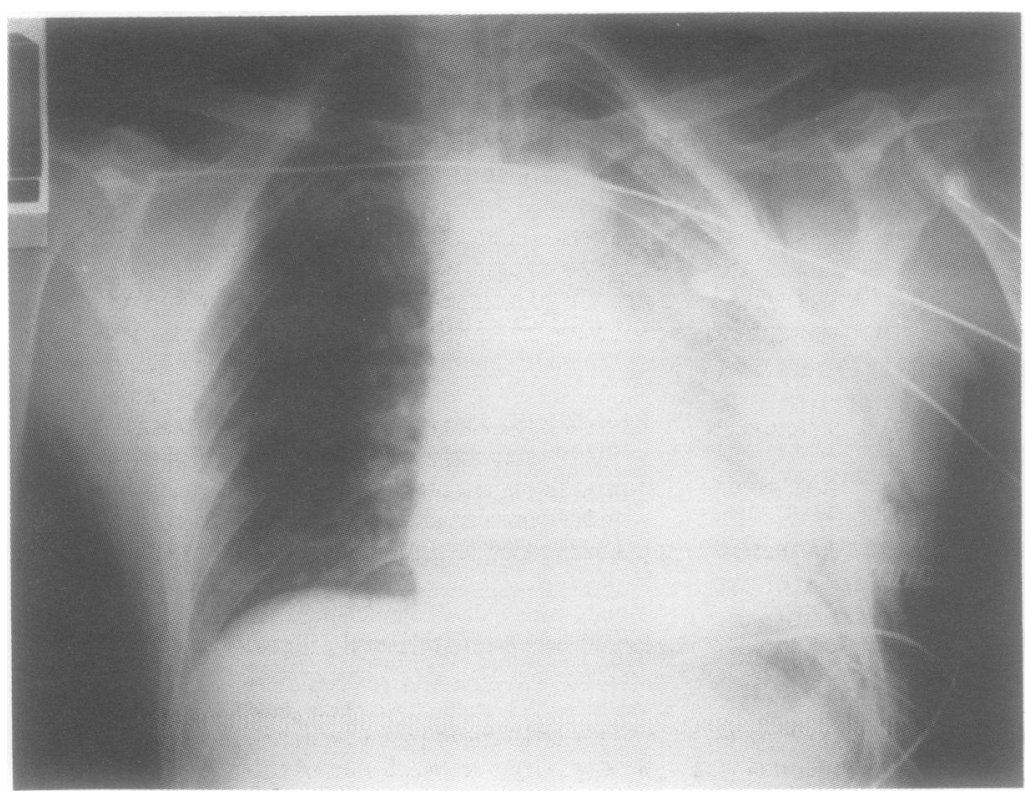

Figure 1 Postoperative chest radiograph showing consolidation of the left lower lobe. tumour in the left upper lobe. Bronchoscopic biopsy demonstrated a giant cell carcinoma. A left upper lobectomy was performed. The inferior pulmonary ligament was divided to allow craniad rotation of the lower lobe. Immediate postoperative recovery was uneventful, but 12 hours after the operation haemoptysis occurred with progressive consolidation of the left lower lobe on the chest radiograph (fig 1). There was a suspicion of lower lobe torsion with rotation of the bronchial stump on bronchoscopic examination. A repeat thoracotomy was urgently performed and torsion of the lower lobe was corrected. However, haemorrhagic necrosis of the lung necessitated completion pneumonectomy. On awakening the patient had a left hemiplegia with a massive cerebral infarction on the CT brain scan (fig 2). His neurological condition deteriorated and he died three days later as a result of raised intracranial pressure. There were no cardiac rhythm disturbances. Post mortem examination revealed congestion of the right lung, liver, and spleen. The carotid arteries were normal. Thrombosis of the left renal artery was present with renal infarction. A thrombotic infarction with massive oedema was found in the right hemisphere in the area of the anterior and middle cerebral artery.

\section{Survey}

A questionnaire on the occurrence of torsion following lobectomy was sent to 12 thoracic surgical departments in Flanders. Responses were received from seven thoracic surgeons, five of whom had never seen a post-lobectomy torsion.

Two surgeons reported having seen torsion following lobectomy. The first involved a middle lobe after right upper lobectomy for tuberculosis. This was initially managed conservatively but several months later a completion pneumonectomy was performed for a carcinoma in the right lower lobe. Postoperative recovery was uneventful. The second case was a torsion of the lower lobe after left upper lobectomy for an endobronchial lipoma. Bronchoscopy was performed for presumed atelectasis during which cardiovascular collapse occurred. The patient died two days later of multiorgan failure.

\section{Discussion}

Pulmonary torsion after lobectomy is a rare occurrence and most cases have been reported during the last two decades. ${ }^{1-10}$ It may occur more frequently than previously suggested, however, as $30 \%$ of the thoracic surgeons in one survey ${ }^{1}$ had seen at least one case of lobar torsion, mostly involving the middle lobe after right upper lobectomy.

Eleven other cases of lobar torsion after pulmonary resection are documented. ${ }^{2-10}$ Again, middle lobe torsion occurred most frequently (seven cases) and right upper lobe torsion was never seen. On the left side there was an equal distribution between upper and lower lobes. In only two cases was re-expansion possible; the 


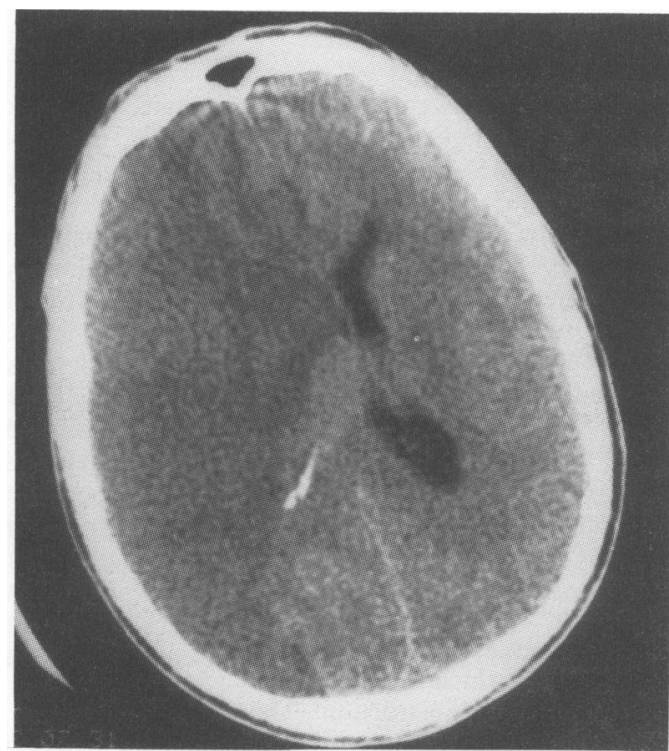

Figure 2 Computed tomographic scan of the brain showing right cerebral infarction with oedema.

remainder underwent resection. Three cases came from the same institution and were the only torsions seen in a group of 1380 lobectomies $(0 \cdot 2 \%) .^{3}$ Only one other case of cerebral infarction complicating pulmonary torsion has been reported. ${ }^{4}$ In this case torsion of the upper lobe occurred after left lower lobectomy for a carcinoid tumour in a Japanese patient. Repeat thoracotomy was performed and showed a $180^{\circ}$ rotation of the upper lobe; the torsion corrected without resection. Afterwards the patient developed a right hemiplegia with left cerebral infarction visible on the CT brain scan. Emboli from a thrombus in the pulmonary vein were thought to be responsible for the cerebral infarction. The same is true for our case where emboli were also found in the renal artery on post mortem examination.

Early intrapericardial clamping of the pulmonary veins before attempting to reposition the lobe may have prevented this complication. This is supported by the observation of Schuler who found a fresh thrombus in the pulmonary vein while resecting the middle lobe after torsion. ${ }^{5}$

A high index of suspicion is necessary for early diagnosis of post-lobectomy torsion. The most important clinical signs are progressive deterioration with increasing blood loss, bronchorrhoea, and haemoptysis. A radiograph and CT scan of the thorax show progressive consolidation of the involved lobe and brochoscopy may reveal rotation of the bronchial tree, especially when the remaining bronchial stump is not found in its expected position.

Predisposing factors for pulmonary torsion are a complete transverse fissure without parenchymal bridges between contiguous lobes, a long free pedicle, incomplete expansion, pleural effusion, and complete division of the inferior pulmonary ligament after upper lobectomy. It is usual practice to allow craniad rotation of the lower lobe after upper lobectomy. However, as this may induce pulmonary torsion, only partial transection of the ligament is advised. When two lobes remain after a lobectomy, prevention of post-lobectomy torsion by sutures or the use of fibrin adhesive is necessary.

We thank all the thoracic surgeons who participated in our survey.

1 Wong PS, Goldstraw P. Pulmonary torsion: a questionnaire survey and a survey of the literature. Ann Thorac Surg 1992;54:286-8.

2 Kelly MV, Kyger ER, Miller WC. Postoperative lobar torsion and gangrene. Thorax 1977;32:501-4.

3 Shirakusa T, Motonaga R, Takada S, Sakuragi T, Dan K. Lung lobe torsion following lobectomy. Am Surg 1990; 56:639-42.

4 Inoue H, Kanabuchi K, Ogawa J, Koide S, Kawada S, Shohtsu A. Torsion after left lower lobectomy and cerebral infarction following the exploratory thoracotomy: a case report. Kyobu Geka 1990;43:724-7.

5 Schuler JG. Intraoperative lobar torsion producing pulmonary infarction. I Thorac Cardiovasc Surg 1973; 65: 951-5.

6 Kucich VA, Villarreal JR, Schwartz DB. Left upper lobe torsion following lower lobe resection. Chest 1989;95:

7 Livaudais W, Cavanaugh DG, Geer TM. Rapid postoperative thoracotomy for torsion of the left lower lobe: case report. Milit Med 1980;145:698-9.

8 Mullin MJ, Zumbro GL, Fishback ME, Neslon TG. Pulmonary lobar gangrene complicating lobectomy. Ann Surg 1972;175:62-6.

9 Pinstein ML, Winer-Muram $\mathrm{H}$, Eastridge C, Scott $\mathrm{R}$ Middle lobe torsion following right upper lobectomy. Radiology 1985;155:580.

10 Weisbrod GL. Left upper lobe torsion following left lingulectomy. f Can Assoc Radiol 1987;38:296-8. 